Chapter 11

\title{
Thermal Transport in Semiconductor Nanowires
}

\author{
J. Anaya, J. Jiménez and T. Rodríguez \\ Additional information is available at the end of the chapter \\ http://dx.doi.org/10. 5772/52588
}

\section{Introduction}

Since the late 1990's semiconductor NWs research is in expansion because of the huge number of potential applications of these one dimensional structures. This research effort is given expression as a vast number of research articles. Semiconductor NWs are the building blocks of advanced nanodevices; e. g. field effect transistors, interconnects, heterostructured devices, as vertically oriented nanoscale laser diodes and HBTs, thermoelectric converters, photovoltaic devices, and sensors [1-5].

The thermal transport in NWs is receiving a great deal of attention, because it constitutes a critical issue for the performance and reliability of NWs based devices. Because of the size reduction a large power density needs to be dissipated, especially in devices operating at high current and power densities; which induces a non negligible Joule heating; therefore, the control of the thermal conductivity is crucial to the thermal extraction from the active parts of these devices [6]. On the other hand, bulk semiconductors are usually good thermal and electrical conductors, which limits their potential performance in thermoelectric devices; however, it was demonstrated that surface engineered semiconductor NWs behave essentially as thermal insulators [2], being at the same time good electrical conductors, this means that semiconductor NWs can have a large themoelectric figure of merit [2,7-9]; which can make them very good thermoelectric materials for waste heat recovery and power generation over a large temperature range.

The experimental measurement of the thermal conductivity at the nanoscale is very challenging [11-13], with very few available contrasted measurements. In this context of lack of experimental results, the development of confident models for describing the thermal conductivity of semiconductor NWs is very important in order to design the thermal management of devices, or the thermoelectric power conversion. All this has created the need for a great modeling effort permitting the design of nanodevices in which the thermal transport plays a capital role. 
We present herein a review of the thermal transport in semiconductor NWs. The main experimental achievements are presented, both smooth and surface engineered NWs, also alloyed NWs are considered; these data evidence the relevance of the thermal transport problem in these one dimensional structures. These experimental data, though scarce, permit a certain degree of contrast of the theoretical models. According to this we present a critical overview of the main theoretical approaches to the problem of the thermal conductivity of semiconductor NWs following a historic sequence. Along this way, we review a large number of models, among others the classical approaches based on the Callaway formalism, atomistic models based on molecular dynamics simulations, or the hydrodynamic approaches. One revises the contribution of the different resistive phonon scattering processes. Also, the role of normal scattering processes is discussed, as well as the recent consideration of the scattering by optic phonons. Special attention is paid to the boundary scattering which is the main cause for the thermal conductivity suppression in semiconductor NWs. The role of engineered surfaces is also discussed.

The strengths and weakness of the main models are discussed. Finally, we introduce a predictive approach extended to the full temperature range, based on the Callaway-Holland formalism, which permits an excellent fitting to the experimental data available. Using this approach empiric relations are deduced for the case of Si NWs, which permit to extract the thermal conductivity for a wide range of diameters and temperatures, which presents an unquestionable interest for practical purposes related to the nanodevice design and thermal managing.

\section{Experimental approach to the thermal transport in semiconductor nanowires}

The measurement of the thermal conductivity at the nanoscale is a challenging experiment. The first measurements of thermal transport of semiconductor NWs were carried out by D. Li et al. [11,13], they measured the thermal conductivity of a few individual Si NWs grown by the vapour liquid solid (VLS) method. The experiments were performed in a microdevice specially designed for measuring the thermal properties of one-dimensional structures [10]. This device, called thermal bridge, consists of a nanowire suspended in between two plates of platinum, one of them acting as the heater, and the other as the thermometer. To guarantee accurate measurements with this device, the junction between the NW and the platinum plates must have an ideally null thermal resistance [10,12].

The results obtained by D. Li et al. [11] showed a strong suppression of the thermal conductivity of the Si NWs as compared with bulk Si (Figure 1-A). Furthermore, a dependence of the thermal conductivity with the NWs diameter was revealed; the smaller the NW diameter the lower the thermal conductivity. On the other hand, the experimental data suggest a change in the temperature dependence of the thermal conductivity of Si NWs for diameters below $25 \mathrm{~nm}$; the experimental data give a linear dependence with $\mathrm{T}$ for the thermal conductivity of a NW with $22 \mathrm{~nm}$ diameter, in which phonon confinement cannot be neglected [15], with respect to the dependence observed for larger diameter NWs, which follow a behavior similar to the bulk(Figure 1-B). 

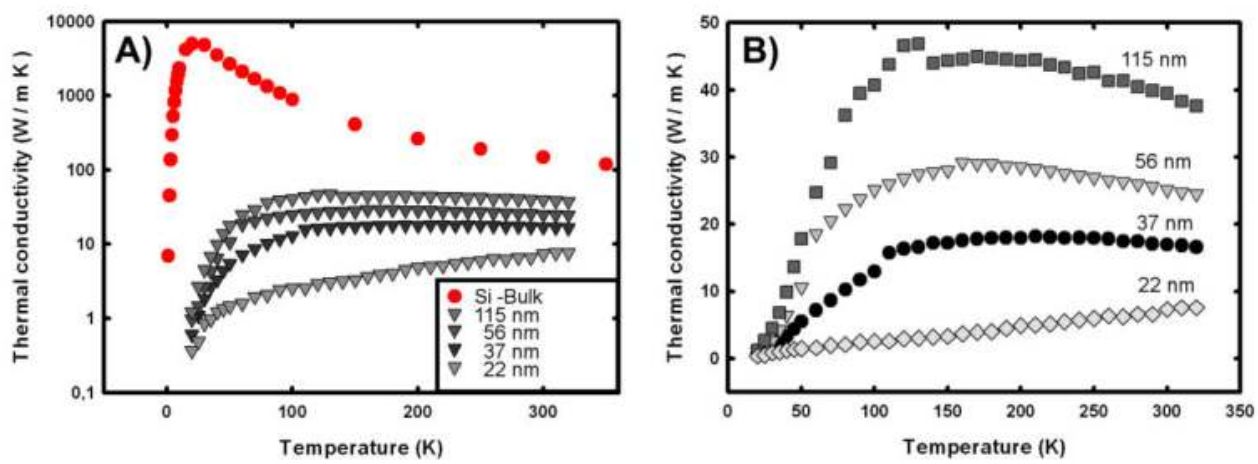

Figure 1. Experimental thermal conductivities as a function of temperature for bulk Si [14], and NWs with different diameters [11] (A), thermal conductivity of NWs in an expanded scale (B) data from [11].

Using the thermal bridge device developed by S. Li et al., the thermal conductivity of other semiconductor NWs has been measured [13,16-20]. All the NWs measured, independently of their nature display a dramatic reduction of the thermal conductivity with the diameter, clearly revealing that the thermal conductivity of NWs is limited by the NWs dimensions. M. C. Wingert et al. carried out thermal conductivity measurements on Ge NWs [20], showing that below a critical diameter of the NW, the thermal conductivity follows a linear dependence with temperature (Figure 2), in agreement with the behavior of the $22 \mathrm{~nm}$ Si NW measured in $[11,13]$. Therefore, this phenomenon can be considered as an intrinsic property of the NWs with reduced dimension, suggesting changes in the phonon transport when phonon confinement effects start to be relevant.

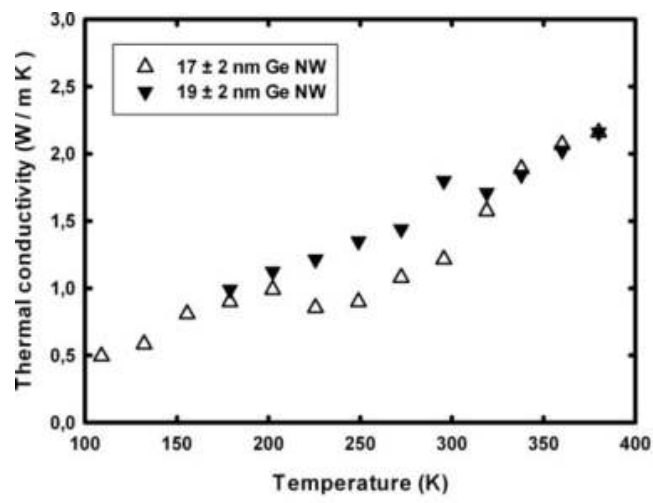

Figure 2. Thermal conductivity of two Ge NWs with diameters below the phonon confinement threshold, showing a linear dependence with temperature [20] 
The thermal conductivity of NWs is not only suppressed by the reduced diameter, but, A. Hochbaum et al. showed that the surface corrugation of the NWs is a major issue for the reduction of the thermal conductivity [2]. Using a similar microdevice to the one described in [10], they measured the thermal conductivity of Si NWs fabricated by an aqueous electroless etching method. These NWs present rough surfaces; in particular, the mean roughness height was estimated near $5 \mathrm{~nm}$, with a lateral roughness correlation length of a few nanometers [2]. The experimental thermal conductivities of two NWs of $115 \mathrm{~nm}$ diameter and comparable length, but different surface roughness are represented in Figure 3, showing a five times reduction in the thermal conductivity for the rough NW. Furthermore, in NWs with engineered surfaces, one can expect that the thermal conductivity could be reduced down to almost the amorphous limit [2].

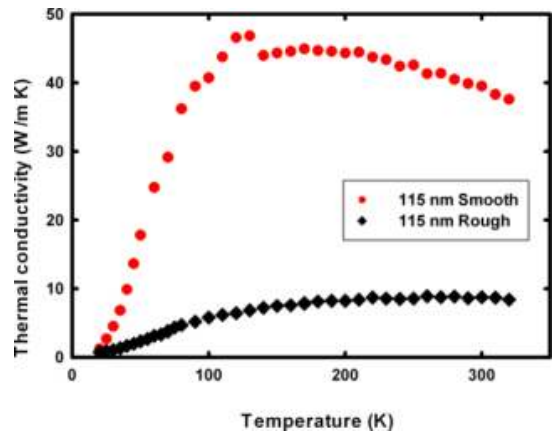

Figure 3. Measured thermal conductivities of two Si NWs of $115 \mathrm{~nm}$ diameter, one of them smooth [11] and the other rough [2].

More recently, Y. Park et al. [21] carried out an experiment similar to that performed in [2], but using rough Si NW grown by an VLS method, by which they grew Si NWs with a mean roughness height close to $10 \mathrm{~nm}$, even greater than the NWs used in [2]. However, the thermal conductivity was not so severely suppressed as it did for the electroless etched NWs prepared in [2]. This illustrates that the reduction of the thermal conductivity is not only propitiated by the roughness height, but as J. Lim et al. have recently shown it is the combination of the roughness height and its lateral correlation length, defined as the mean distance between surface hills/depressions, that limits the thermal conductivity in rough NWs [22]. They measured the thermal conductivity of several Si NWs with different surface morphologies, showing that the distance between surface hills/depressions plays a crucial role in the decrease of the thermal conductivity. The SEM images of the NWs measured in [21] compared to the SEM images of the NWs measured in [2], clearly show that the distance between hills/depressions of the NWs of reference [21] are significantly longer than for the NWs of reference [2], which should account for the lower thermal conductivity reduction observed by Lim [22] with respect to the strong reduction reported by Hoschbaum, despite the higher value of the mean roughness height measured in [21]. 
$\mathrm{H}$. Kim et al. showed the effect of composition on the thermal conductivity of semiconductor NWs. They measured the thermal conductivity of alloyed $\mathrm{Si}_{1-x} \mathrm{Ge}_{\mathrm{x}} \mathrm{NWs}$ with a small Ge fraction [23]. Even for very diluted alloys the thermal conductivity of the NWs showed a dramatic reduction compared to pure $\mathrm{Si} N \mathrm{NW}$. Thereafter, $\mathrm{H}$. Kim et al. grew rough $\mathrm{Si}_{1-\mathrm{x}} \mathrm{Ge}_{\mathrm{x}} \mathrm{NWs}$ and showed the combined effect of the alloy composition and the surface roughness [24], which permits additional reductions of the thermal conductivity; thus, opening almost unlimited possibilities for designing nanodevices by its thermal conductivity, which is very attractive for thermoelectric devices. These results are summarized in Figure 4, where the experimental values for a smooth $\mathrm{Si} \mathrm{NW}$, and a smooth $\mathrm{Si}_{1-\mathrm{x}} \mathrm{Ge}_{\mathrm{x}} \mathrm{NW}$ of comparable diameter and a very low $\mathrm{Ge}$ fraction $(x=0.004)$ are shown. The effect of the roughness is summarized in two NWs of the same composition and diameter but differing only by the surface roughness.

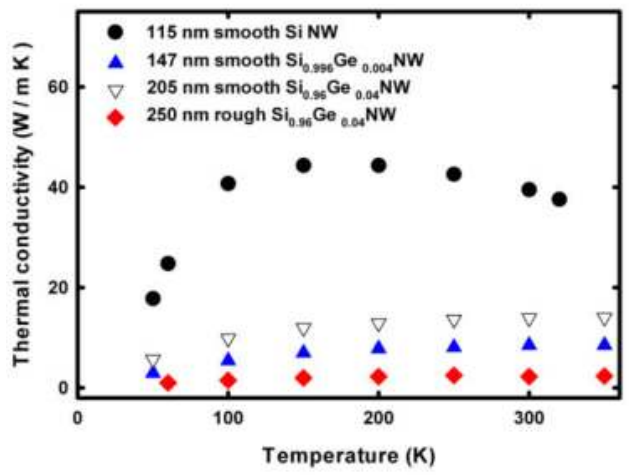

Figure 4. Thermal conductivities of a smooth Si NW from [11] (circles), and alloyed SiGe NWs, smooth NWs data from [23] (triangles) and rough NWs data from [24] (diamonds).

In a recent work, Y. Choongho reported additional thermal conductivity data of $\mathrm{Si}_{1-\mathrm{x}} \mathrm{Ge}_{\mathrm{x}} \mathrm{NWs}$ for several compositions [18]; evidencing the relevant role played by the composition, but one cannot be conclusive about the composition bowing, because of the diameter dispersion.

\section{Theoretical models}

Before the first experimental measurement of the thermal conductivity in a semiconductor NW, a few theoretical approaches already pointed to a thermal conductivity decrease in low dimensional structures. Five years early with respect to the first experimental measurements of NW thermal conductivity, A. Balandin et al. in a pioneering work on the effect of low dimensionality on the thermal transport, presented a theoretical analysis of the lattice thermal conductivity of a semiconductor quantum well [25]. This analysis was built up on the basis that the spatial confinement involves a reduction of the phonon group velocities, therefore, of the phonon relaxation rate. This thermal transport reduction makes evident the increased 
relevance of the resistive phonon processes in reduced dimension structures. They calculated the thermal conductivity of Si quantum well [25] making use of a reduced Callaway formalism [26], in which only considered the resistive processes given by the Umpklapp scattering, the boundary scattering, and the processes related to the impurity scattering. A detailed description of the Callaway model and the different scattering mechanisms is addressed in paragraph 4. 2. The model proposed in [25] revealed that the thermal conductivity of a Si nanostructure must be substantially reduced with respect to its bulk counterpart. Shortly after, Zou and Balandin applied the same formalism to the study of the thermal conductivity of Si NWs [27]. They modified the simple model described on [25] under the basis of a phonon redistribution by the NW boundaries, which implies a modification of the reduced Callaway expression used in the previous work. On the other hand, the thermal conductivity dependence with the surface roughness of the NWs was also added. The scattering processes considered were a modification of the Umpklapp scattering, the electron-phonon scattering, and a modified boundary scattering taking account of the effect of roughness, expressed in terms of the fraction of specularly to diffusively reflected phonons at the NW boundary [28]. With those modifications, the thermal conductivity of a Si NW with $20 \mathrm{~nm}$ diameter was calculated, showing a great dependence of the thermal conductivity reduction with the surface characteristics. Nevertheless, even in the case of the maximum surface contribution, the thermal conductivity calculated was higher, $14 \mathrm{~W} / \mathrm{mK}$ at $300 \mathrm{~K}$ for $20 \mathrm{~nm}$ diameter, near twice, than the experimental value reported for a $22 \mathrm{~nm}$ diameter $\mathrm{Si} \mathrm{NW}$ $[11,13]$. In a different approach, A. Voltz et al. made use of molecular dynamics simulations in thin Si NWs with diameters below $5 \mathrm{~nm}$ [29], reaching the same result of a dramatic reduction of the thermal conductivity.

The first experimental data about the thermal conductivity of Si NWs opened the possibility to contrast the theoretical models [11,13], allowing the possibility of developing predictive tools regarding the thermal conductivity of NWs. As mentioned above, the experimental measurements evidenced a strong dependence with the NWs morphology, including diameter and surface topography; as a matter of fact, the construction of a model capable of predicting the thermal conductivity of semiconductor NWS becomes a necessary tool for the design of nanodevices in which the thermal transport becomes a critical issue for performance and reliability. N.Mingo build up a model based on a modification of the ballistic phonon transport in a suspended NW [30]. The model takes account of the scattering events that undergo the phonons in a real semiconductor, and requires the calculation of the complete phonon dispersion relations in the NW. The phonon scattering considered by Mingo, as it was the case for the Balandin models [25,27], are the resistive processes given by Umpklapp, boundary, and impurity scattering mechanisms. At difference of the models reported on references [25,27], where exact expressions for the Umpklapp scattering were used, Mingo introduced two parameters which the values are obtained from the adjustment of their model to the thermal conductivity of bulk Si. [30] These parameters are fixed for the calculation of the thermal conductivity of the NWs; therefore, it can be considered as a free of parameters model. Unfortunately, this model underestimates the thermal conductivity value with respect to the experimental data, requiring of an additional adjustment parameter concerning the boundary scattering; this parameter is defined as an effective diameter, greater than the 
diameter of the NWS experimentally measured in the SEM [30]. Thereby, using effective diameters up to $30 \%$ higher than the experimentally measured ones, Mingo obtained a satisfactory fitting to the experimental values reported for NWs with diameters above the limit for phonon confinement. [30,31] On the other hand, Mingo showed that a reduced Callaway model as the one developed by Balandin et al. $[25,27]$ can give a good fitting to the experimental values if one lowers the cut-off temperature with respect to the bulk [30].

Those models only deal with the resistive scattering processes; this means that the contribution of the normal three-phonon events is neglected. However, it is well known that these processes contribute to the thermal transport resistance through the Herring mechanism [32]. Y. Chen developed a model based on Monte Carlo simulation considering normal and resistive processes [33]. Making use of a complex genetic algorithm and resorting to two free adjusting parameters, they showed a good fitting to the experimental data in the low temperature range $(\mathrm{T}<120 \mathrm{~K})$, but overestimated the thermal conductivity for higher temperatures. Once again the behavior exhibited by the $22 \mathrm{~nm}$ smooth Si NW was not reproduced by the model, which responded reasonably well above this limit.

Coming back to the first models developed by Zou et al. [27], M. Huang et al. carried out a more detailed treatment of the boundary scattering [34], presenting it as a rational function of the fraction of specularly reflected phonons at the boundary instead of the linear dependence originally used in [27]. Despite of being a model with a more realistic treatment of the boundary scattering the result was not satisfactory. On the other hand, they showed that not only the sound velocities decreased due the reduced diameter of the NWs, but the Debye temperatures had also be lower [34]; which, should account for the good agreement to the experimental data reached by Mingo when using cut-off temperatures lower than the bulk silicon cut-off temperatures in the Callaway model [30].

L. Liang et al. used a simple approach in which they established direct relations between the bulk semiconductor melting point and the nanowire melting point [35]; furthermore, they introduced an exponential expression for describing the boundary scattering due to surface roughness. This simple approach was able of predicting the thermal conductivity at $300 \mathrm{~K}$ for all the NWs measured in [11], even for the problematic $22 \mathrm{~nm}$ Silicon NW. The anomalous behavior of this thin NW, and thus of those NWs with diameter below the limit for phonon confinement,[11,20] was studied by P. Murphy et al. [36]. In contrast to previous works, they argued that in a strongly disordered surface not all the phonons are equally scattered, being these events dependent on the frequency of the incident phonons. Based on these assumptions, they developed a model splitting the behavior of the phonons in two different contributions, determined by the magnitude of the component of their wave vectors in the direction perpendicular to the NW axis. The behavior is nearly ballistic for phonons with a small transverse wave vector component, while for those with a large transverse wavevector component the phonon scattering was assumed to be purely diffusive, with a mean free path of the order of the NW diameter. Under these assumptions, they showed that for the low temperature range, the calculated thermal conductivity approaches to a linear dependence with the temperature for decreasing NW diameters [36]; further showing that the change from the $\mathrm{T}^{3}$ law for bulk and thick NWs to the linear dependence for the 
thinner NWs occurs between $25 \mathrm{~nm}$ and $20 \mathrm{~nm}$ in diameter, as the experimental measurements demonstrate $[11,20]$.

Z. Tian et al. suggested that the role of the optical phonons must be taken into account for nanostructures [37]. Making use of the phonon-phonon scattering description derived by A. Ward et al. [38], they carried out the calculation of the thermal conductivity contribution of each phonon branch, showing that the optical phonon contribution to the thermal conductivity in NWs can represent near $20 \%$ of the total. Under the assumption of a diffusive regime for the boundary scattering, therefore in the Casimir limit [28], they calculated the thermal conductivity of smooth Si NWs in a good concordance with the experimental values. In a previous work, M. Kazan et al. had already considered the optical phonon contribution [39]. They showed how the consideration of the optical phonon decay into acoustic phonons in the frame of the Callaway formalism can cause an improvement in the prediction of the thermal conductivity of bulk Ge. Subsequently M. Kazan et al. applied this method to smooth Si NWs [40]. It should be noted, the Callaway formalism was conceived without the contribution of the optical modes [41-43]; although, it is implicitly taken into account because of the increase of the cut-off temperatures necessary for fitting the experimental data [44]. Therefore, in the modified Callaway model introduced in [40],which explicitly considers the optical contribution, the cut-off temperatures are lower than the cut-off temperatures used when applying the Callaway formalism without considering the optical phonon contribution in a explicit way. Finally, working in the Casimir limit for boundary scattering and making use of a large number of adjustment parameters, they obtained a very good fitting to the thermal conductivity of Si NWs [40].

All those models, which give good fittings to the experimental values of the thermal conductivity for Si NWs work in the diffusive limit for the boundary scattering; this means that one cannot further reduce the thermal conductivity due the boundary scattering when considering rough surfaces. Thereby, when A. Hotchbaum et al. measured experimentally the thermal conductivity of rough Si NWs showing a great reduction in the thermal conductivity of rough NWs compared with the smooth Si NWs [2], none of those models could fit the experimental values.

F. Alvarez et al. showed that the thermal conductivity of semiconductor NWs can be described making use of an analogue hydrodynamic model for the phonon flux [45]. By solving the Guyer-Krumhansl equation for the phonon flux in a NW [46, 47], they reached a very simple expression for the thermal conductivity depending on the bulk thermal conductivity, the dimension of the NWs, and the fraction of specularly reflected phonons at the boundary [48]. In a further study, A.Sellito et al. showed that in a hydrodynamic framework, the roughness of the boundaries may cause a local inversion of the phononic flux, thus extra limiting the thermal conductivity of the NW [49]. This result is consistent with a previous work by A. Moore, who showed how the surface roughness of a NW may cause backscattering events resulting in a more aggressive limitation of the thermal conductivity than the diffusive limit considered in the previous models [50]. On this basis, A. Sellito et al. [51] returned to the hydrodynamic model described in [48], which gave a simple expression for the thermal conductivity, and added the contribution of the backscattering events. With this 
modification, they attained a good fitting to the thermal conductivity of the smooth Silicon NWs below $150 \mathrm{~K}$ [51], besides, obtaining values for the thermal conductivity of the rough NWs close to those reported in reference [2] in the same temperature range.

The effect of the surface roughness in the boundary scattering was studied by P. Martin et al. using perturbation theory [52]; the perturbation due to surface roughness was introduced in the Hamiltonian; also, the existence of a thin layer of native $\mathrm{SiO}_{2}$ layer surrounding the NW was considered. This model shows values close to the experimental measurements for smooth Si NW, but without a satisfactory fitting to the experimental data of reference [11]. More interesting is the capability shown by the model when one evaluates the thermal conductivity of rough NWs, insomuch as the values are close to those measured by Hochbaum et al. in [2]. On the other hand, they showed that the effect of the boundaries in the thermal conductivity reduction increases the more the smaller the diameter [52]; which suggests a dependence of the thermal conductivity with the diameter and the mean roughness height $(\eta)$, following the relation $k \propto(D / \eta)^{2}$.

Other approaches making use of molecular dynamics simulations intended to assess the effect of the roughness in the phonon transport $[53,54]$, showing that the reduction of the thermal conductivity is strongly dependent on the morphology of the surface. On the other hand, D. Donadio using atomistic simulations [55], suggested that this reduction is due to the flattened phonon dispersion curves causing non-propagating phonon modes. Afterwards, M. Luisier on the basis of the atomistic quantum transport corroborated these results [56]. Moreover, he suggested that there is no need of large mean roughness height to enter in the pure diffusive limit in which the mean free path (MFP) is comparable to the diameter of NW; thus, giving an explanation of why the models that work in this hypothesis for the smooth NWs measured in [11] were getting a good fitting.

J. Shadhu et al developed a model in which they introduced different frequency-dependent regimes for the scattering rates and a complex analysis of the roughness contribution [57]. They found that the correlation length plays a major role in the reduction of the thermal conductivity. Short correlation lengths leads to a larger number of phonons suffering diffusive surface scattering events; even more, a large roughness correlation length implies that only the phonons with long wavelengths, and thus phonons that do not significantly contribute to the thermal transport, are scattered by the surface. The correlation length also determines the incident angles for which the phonons suffer a scattering event. On the other hand, they indicate that the short wavelength modes are strongly attenuated in rough boundaries with short correlation lengths, resulting in a MFP for these phonons shorter than the NW diameter, which can be interpreted as a breakdown of the Casimir limit. With this complex model, they obtained a very good fitting to the experimental thermal conductivities for both rough and smooth Si NWs. Even more, the results achieved in this work are consistent with the recent work of J. Lin that demonstrated experimentally the importance of the correlation length together with the roughness mean height on the thermal conductivity reduction in semiconductor NWs [22].

The idea of a breakdown in the Casimir limit for rough NWs was previously pointed out, but without a physical explanation accounting for it. When C. Guthly et al. measured the 
thermal conductivity of rough gallium nitride NWs [17], they showed that a very good fitting to the experimental values can be achieved using the Callaway model with a modified boundary scattering. Taking into account that the diffusive limit, characterized by a phonon MFP equal to the diameter of the NW, cannot take account of the roughness effect, they used the MFP as an adjusting parameter. A very good fitting to the experimental values was achieved for values of MFP below the NW diameter, therefore, below the Casimir limit. The result of this paper, together with the confirmation of the violation of the Casimir limit for rough NWs [57], allow the use of simple models based on the Callaway formalism for calculating the thermal conductivity of rough NWs, making use of a reduced effective diameter for the boundary scattering.

When considering alloyed NWs, e. g. $\mathrm{Si}_{1-x} \mathrm{Ge}_{x} \mathrm{NWs}$, the thermal conductivity modeling must address the effect of the alloy scattering, besides all the above mentioned scattering terms. $\mathrm{N}$. Yang et al. carried out a first approximation in NWs composed of a mixture of Si isotopes making use of a molecular dynamic simulation [58]. They showed a large reduction in thermal conductivity, due to the isotopic scattering, with a maximum reduction for a composition of $50 \%$ between the constituent specimens. Afterwards, J. Chen et al. performed an equivalent molecular dynamics simulation on $\mathrm{Si}_{1-x} \mathrm{Ge}_{x}$ NWs [59], showing a strong dependence of the thermal conductivity on the composition of the NW. Furthermore, they found that the thermal conductivity is severely reduced even for small fractions of Ge, what was later on experimentally demonstrated [23]. They estimated a reduction in the thermal conductivity of $50 \%$ with respect to pure Si NWs for a Ge fraction of only $5 \%$; however, one experimentally observes a much greater reduction, even for a smaller fraction of Ge in the NW [23]. In another attempt to modeling the thermal conductivity of $\mathrm{Si}_{1-x} \mathrm{Ge}_{\mathrm{x}} \mathrm{NWs}, \mathrm{Z}$. Wang [60] suggested a model on the basis of the previous works made by N. Mingo for Si NWs [30]. They assumed that all the scattering relaxation times, except the impurity relaxation time, can be viewed as independent processes for Si and Ge; therefore, they were added according to the Mathisessen's rule weighted by the composition, while the impurity scattering related to the alloy, is assumed to depend of the composition by a quadratic bowing function. In this way, they obtained a simple model able to achieve a prediction of the thermal conductivity of the order of magnitude of the experimental values obtained in [23]. Unfortunately, although they estimate thermal conductivity values of the order of magnitude of the experimental results, the fitting to the experimental results was not satisfactory, especially in the low temperature range.

Additional work is still needed to supply a good model accounting for the experimental thermal conductivity of $\mathrm{Si}_{1-\mathrm{x}} \mathrm{Ge}_{\mathrm{x}} \mathrm{NWs}$, and in general of alloyed, or doped NWs. If one observes the historical development of the models elaborated for describing the thermal conductivity of pure semiconductor NWs, one can see that the major advances were achieved once reliable experimental data were available. Very recently, new measurements of $\mathrm{Si}_{1-x} \mathrm{Ge}_{\mathrm{x}}$ NWs have been published by Y. Choongho in a wide range of compositions [18], suggesting that more new models concerning alloyed NWs will arrive in the next future. 


\section{A Predictive model for the thermal conductivity of NWs}

\subsection{Preliminary remarks}

As discussed in the previous paragraph different approaches have been attempted for the description of the thermal conductivity of semiconductor NWs. The measurement of the thermal conductivity at the nanometer scale is a rather complex experimental exercise; usually, the experimental data are hindered by the incertitude introduced by the NW/ heat sink contact. Therefore, the experimental data providing the thermal conductivity of NWs are scarce, and only a few experiments have supplied reliable thermal conductivity data; these data have been used to check the validity of the thermal transport models allowing a significant advance of the modeling formalisms. Concerning the different approaches used for modeling the thermal conductivity of NWs we have already mentioned that, some of them attained a good fitting to the experimental data using complex calculations, some other required of too many fitting parameters; while some other provided a good fitting in a reduced temperature range. The lack of generalized experimental results is a serious problem for both the design of thermoelectric devices based on NWs, and the thermal management of NW based devices. Therefore, a comprehensive model giving a predictive estimation of the thermal conductivity with the factors that contribute to limit it, e. g. the NW diameter, and the surface roughness, would be of high technological interest. On the other hand, experimental data are restricted to temperatures below room temperature, the data at higher temperatures are not available; however, this information is required for many applications that can operate at higher temperatures. We present herein a predictive model based in the well-known Callaway-Holland formalism that substantially simplifies the estimation of the thermal conductivity, provides an excellent fit to the experimental data, and can be used as a tool for all the technical situations in which the thermal conductivity of NWs is required for the design of NW based devices.

\subsection{The Callaway-Holland formalism}

The Callaway-Holland formalism has been successfully used for the description of the thermal conductivity of bulk semiconductors [40,42,61]; therefore, it must be conveniently adapted to the nanoscale requirements. It is well known that one of the main consequences of the reduced dimension of the NWs is the appearance of quantum confinement effects; in particular, the phonon confinement is relevant for diameters below 20-25 nm [15]. Many of the applications with NWs are built up with NWs with diameters above this phonon confinement limit; therefore, for these NWs one can reasonably assume a bulk like behavior.

First, we will introduce the main features of the Callaway-Holland formalism, initially developed for the low temperature range $[41,43]$. Despite the original limitation in temperature, the formalism was demonstrated to be a very useful tool in the calculation of thermal conductivity of semiconductor materials over the full range of temperatures, by the addition of some modifications.

The thermal conductivity is calculated by solving the Boltzmann equation 


$$
\left(\frac{\partial N}{\partial t}\right)_{\mathbf{c}}-\mathbf{c} \nabla T \frac{d N}{d T}=0
$$

where $\mathrm{N}$ is the phonon density, and $c$ the group velocity. In the presence of a small temperature gradient, $\nabla T$, the deviation from equilibrium of the phonon mode k damps out exponentially, and the phonon population decay can be described by a specific relaxation time.

$$
\left(\frac{\partial N}{\partial t}\right)_{\mathbf{c}}=\frac{N^{0}-N}{\tau(\mathrm{k})}
$$

$N^{0}$ is the Planck distribution function, and $\tau(\mathrm{k})$ is the relaxation time of the k mode. Callaway split out this expression in order to taking account of the contributions of both normal and resistive phonon processes, giving the following expression for the variation of the phonon density.

$$
\left(\frac{\partial N}{\partial t}\right)_{\mathbf{c}}=\frac{N(\boldsymbol{\lambda})-N}{\tau_{N}}+\frac{N^{0}-N}{\tau_{R}}
$$

Where $N(\lambda)$ is the shifted Planck distribution function towards which the Normal processes approach [62]; it can be expressed in a first approximation as:

$$
N(\boldsymbol{\lambda}) \neg N(0)+\lambda\left(\frac{\partial N}{\partial \boldsymbol{\lambda}}\right)_{0}=N^{0}+\frac{\lambda \mathbf{k}}{K_{B} T} \frac{e^{\hbar \omega / K_{B} T}}{\left(e^{\hbar \omega / K_{B} T}-1\right)^{2}}
$$

On the other hand, because a small deviation from equilibrium was assumed one can approximate $\frac{d N}{d T} \approx \frac{d N^{0}}{d T}$, therefore, one can simplify the Boltzmann equation as:

$$
\left(-\frac{\hbar \omega}{K_{B} T} \mathbf{c} \nabla T+\frac{1}{\tau_{N}} \frac{\lambda \mathbf{k}}{K_{B} T}\right) \frac{e^{\hbar \omega / K_{B} T}}{\left(e^{\hbar \omega / K_{B} T}-1\right)^{2}}-\left(\frac{1}{\tau_{N}}+\frac{1}{\tau_{R}}\right)\left(N-N^{0}\right)=0
$$

By introducing a global relaxation time, $\alpha(\mathrm{k})$, the term $N-N^{0}$ of the equation 5 can be expressed as:

$$
N-N^{0}=-\alpha(\mathbf{k}) \mathbf{c} \nabla T\left(\frac{\hbar \omega}{K_{B} T^{2}}\right) \frac{e^{\hbar \omega / K_{B} T}}{\left(e^{\hbar \omega / K_{B} T}-1\right)^{2}}
$$


allowing to transform the Boltzmann equation in the more tractable form:

$$
\left(\frac{\alpha(\mathrm{k})}{\tau_{C}}-1\right)\left(\frac{\hbar \omega}{T}\right) \boldsymbol{c} \nabla T+\frac{1}{\tau_{N}} \lambda \boldsymbol{k}=0
$$

Where $\tau_{C}=\left(\tau_{N}^{-1}+\tau_{R}^{-1}\right)^{-1}$. On the other hand, assuming isotropic media, $\lambda$ must be a constant vector in the direction of the temperature gradient. Thus, one can define a parameter, $\beta$, expressed as $\beta=-\frac{\lambda}{\hbar v^{2}} \frac{T}{\nabla T}$, where $v$ is the speed of sound. Finally, in the acoustic approximation $\mathrm{k}=\frac{c \omega}{v^{2}}$ and substituting this in the reduced Boltzmann equation, one obtains the following expression for the relaxation time:

$$
\alpha=\tau_{C}\left(1+\left(\frac{\beta}{\tau_{N}}\right)\right)
$$

Using this relaxation time, one can estimate the thermal conductivity through the following equation:

$$
\kappa=\frac{1}{(2 \pi)^{3}} \sum_{i} \int_{0}^{2 \pi} \int_{0}^{\pi}\left(\int v_{i}^{2} \alpha_{i}(k) C_{p h}(k)_{i} \cos ^{2} \theta d^{3} \mathbf{k}\right) d \theta d \phi
$$

Where $C_{p h}$ is the phonon specific heat, and $\theta$ the angle formed between the phonon wave vector and the temperature gradient. Making use of the branch separation proposed by Holland [42], and considering that the phonon specific heat is expressed as:

$$
C_{p h}=\frac{d}{d T}\left(\hbar \omega N^{0}\right)=\frac{(\hbar \omega)^{2}}{K_{B} T^{2}} \frac{e^{\hbar \omega / K_{B} T}}{\left(e^{\hbar \omega / K_{B} T}-1\right)^{2}}
$$

The thermal conductivity transforms into the expression

$$
\kappa_{i}=\sum_{i}\left(\frac{v_{i}^{2}}{8 \pi^{3}}\right) \int_{0}^{k_{D}^{i}}\left(\frac{(\hbar \omega)^{2}}{K_{B} T^{2}}\right) \frac{1}{\tau_{C}^{i}}\left(1+\frac{\beta}{\tau_{N}^{i}}\right) \frac{e^{\hbar \omega / K_{B} T}}{\left(e^{\hbar \omega / K_{B} T}-1\right)^{2}} \cos ^{2} \theta d^{3} k
$$

where the subindex refers to the different acoustic branches, the longitudinal and the two transversal branches. The explicit form of the $\beta$ parameter is obtained from the momentum conservation rule in the normal scattering processes; therefore: 


$$
\int\left(\frac{\partial N}{\partial t}\right)_{N} \mathbf{k} d^{3} k=\int\left(\frac{N(\lambda)-N}{\tau_{N}^{i}}\right) \mathbf{k} d^{3} k=0
$$

Replacing the term $N(\lambda)-N$ by their explicit form, previously derived in equation 6 , one arrives to the equation:

$$
\int \frac{e^{\hbar \omega / K_{B} T}}{\left(e^{\hbar \omega / K_{B} T}-1\right)^{2}}\left(\frac{\hbar \omega}{K_{B} T^{2}}\right) c \nabla T(\alpha(\kappa)-\beta) \frac{c \omega}{\tau_{N}^{i} v^{2}} d^{3} \mathrm{k}=0
$$

And making use of $c^{2} \nabla T=v_{i}^{2} \cos ^{2} \theta$ and replacing $\alpha(\mathrm{k})$ by its value given in equation 8 , one obtains:

$$
\int \frac{e^{\hbar \omega / K_{B} T}}{\left(e^{\hbar \omega / K_{B} T}-1\right)^{2}}\left(\frac{\hbar \omega}{K_{B} T^{2}}\right)\left(\tau_{C}^{i}+\left(1-\frac{\tau_{C}^{i}}{\tau_{N}^{i}}\right) \beta\right) \frac{\omega}{\tau_{N} v^{2}} v^{2} \cos ^{2} \theta d^{3} \mathrm{k}=0
$$

From this expression and taking account of the independence between $\beta$ and $\mathrm{k}$ one obtains

$$
\beta=\frac{\int \frac{\tau_{C}^{i}}{\tau_{N}^{i}} \frac{e^{\hbar \omega / K_{B} T}}{\int\left(e^{\hbar \omega / K_{B} T}-1\right)^{2}}\left(\frac{\hbar \omega}{K_{B} T^{2}}\right) \omega \cos ^{2} \theta d^{3} \mathrm{k}}{\int\left(1-\frac{\tau_{C}^{i}}{\tau_{N}^{i}}\right) \frac{1}{\tau_{N}^{i}} \frac{e^{\hbar \omega K_{B} T}}{\left(e^{\hbar \omega K_{B} T}-1\right)^{2}}\left(\frac{\hbar \omega}{K_{B} T^{2}}\right) \omega \cos ^{2} \theta d^{3} \mathrm{k}}
$$

Finally, taking into account that $d^{3} k=\frac{\omega^{2}}{v^{3}} d \omega d \Omega_{k}=\frac{\omega^{2}}{v^{3}} \sin \theta d \theta d \phi d \omega$, and the change of variable $x=\hbar \omega / K_{B} T$, the thermal conductivity can be expressed as:

$\kappa=\sum_{i}\left(\frac{K_{B}^{4} T^{3}}{8 \pi^{3} \hbar^{3} v_{i}}\right)\left\{\int_{0}^{\pi} \int_{0}^{2 \pi} \int_{0}^{\frac{\theta_{i}}{T}}\left\{\tau_{C}^{i} \frac{e^{x}}{\left(e^{x}-1\right)^{2}} x^{4} \cos ^{2} \theta \sin \theta d \theta d \varphi d x\right\}+\right.$

$$
\left.+\left(\frac{\int_{0}^{\pi} \int_{0}^{2 \pi} \int_{0}^{\frac{\theta_{i}}{T}}\left\{\frac{\tau_{C}^{i}}{\tau_{N}^{i}} \frac{e^{x}}{\left(e^{x}-1\right)^{2}} x^{4} \cos ^{2} \theta \sin \theta d \theta d \phi d x\right\}}{\int_{0}^{\pi} \int_{0}^{2 \pi} \int_{0}^{\frac{\theta_{i}}{T}}\left\{\frac{1}{\tau_{N}^{i}}\left(1-\frac{\tau_{C}^{i}}{\tau_{N}^{i}}\right) \frac{e^{x}}{\left(e^{x}-1\right)^{2}} x^{4} \cos ^{2} \theta \sin \theta d \theta d \phi d x\right\}}\right) \int_{0}^{\pi} \int_{0}^{2 \pi} \int_{0}^{\frac{\theta_{i}}{T}}\left\{\frac{\tau_{C}^{i}}{\tau_{N}^{i}} \frac{e^{x}}{\left(e^{x}-1\right)^{2}} x^{4} \cos ^{2} \theta \sin \theta d \theta d \phi d x\right\}\right\}
$$


Were the integral limits $\theta_{i}$ are the characteristic temperature cut-offs of each branch.

\subsection{The phonon scattering terms}

The application of the Callaway-Holland formalism to a particular problem in an extended temperature range resumes in the appropriate characterization of the different scattering terms. Therefore, a detailed analysis of the different scattering processes is required.

\subsubsection{Three phonon scattering}

This kind of process is determined by the exclusive interaction between phonons and follows two ways. In the first one, a phonon of wave vector $q_{1}$ combines with a phonon of wave vector $\mathrm{q}_{2}$ given another phonon of wave vector $\mathrm{q}_{3}$. In the second process a phonon of wave vector $\mathrm{q}_{1}$ decays in two phonons of wave vectors $\mathrm{q}_{2}$ and $\mathrm{q}_{3}$ respectively.

$$
\begin{aligned}
& q_{1}+q_{2}=q_{3} \\
& q_{1}=q_{2}+q_{3}
\end{aligned}
$$

In these processes the energy is conserved; but such conservation does not necessarily hold for the momentum. The processes in which the momentum is not conserved are called Umpkklap, in this process the initial and final moment differs by a non zero reciprocal lattice vector, and the resultant phonons are out of the Brillouin zone; the momentum selection rule for these processes reads as:

$$
\begin{aligned}
& q_{1}+q_{2}=q_{3}^{\prime}+G \\
& q_{1}=q_{2}+q_{3}^{\prime}+G
\end{aligned}
$$

Where $\mathrm{q}_{3}$ is the "reflected" phonon of wave vector $\mathrm{q}_{3}{ }_{3}$, and G the reciprocal lattice vector [28]. The phonon wave vector is opposite to $q_{1}$ and $q_{2}$ phonons, resulting in a process resistive to the heat flow.

Peierls proposed for the Umpklapp processes a scattering rate of the form $\tau_{U}^{-1} \propto T^{a} e^{\theta / b T}$ [62], where the values of the parameters $a$ and $b$ have been determined in different ways [62-65]. Klemens showed that in the high temperature range the resistive contribution of the three phonon processes obeys to $\tau_{U}^{-1}=C \omega^{2} T$ [64]. Using the Slack's variant [65] of the Peierls form, one can extend the temperature range, the Slack relaxation time for the U processes is given as $\tau_{U}^{-1}=B \omega^{2} T e^{\theta / 3 T}$. Using this form, Slack established that the thermal conductivities of different pure crystals can be modeled over an extended temperature range giving a good agreement with the experimental values [65]. Furthermore, when the temperature increases, it adopts the same expression as the Klemens relaxation time. Klemens evaluated the constant $C$ as $C=\frac{2 \gamma^{2} \hbar}{M v^{2} \theta}[27,64]$. Where $\gamma$ is the Grüneisen parameter, $M$ the average atomic mass in the crystal, and $v$ the velocity of sound. Finally, we can introduce the Umpklapp relaxation time for each phonon branch as: 


$$
\left(\tau_{U}^{-1}\right)^{i}=\frac{2 \gamma_{i}^{2} \hbar}{M v_{i}^{2} \theta_{i}} \omega^{2} T e^{\theta_{i} / 3 T}
$$

Regarding the Normal processes, it has been claimed that they do not contribute to the thermal resistance $[25,27,30,31,33]$. However, they cannot be neglected because the CallawayHolland formalism requires of the normal process relaxation time [41-43]. Furthermore, the normal processes can contribute indirectly to the thermal resistance by redistributing the phonon population, which can further participate in resistive scattering processes. These can be explained by the Herring mechanism [32], which redistributes the phonon momentum among the different phonon branches. In this mechanism, the relaxation of transverse phonons consists of its interaction with a longitudinal acoustic phonon, resulting in another longitudinal phonon. On the other hand, the relaxation of the longitudinal phonons occurs via two different processes; they decay in two transverse phonons; or a longitudinal phonon arises from two transverse phonons. Taking account of these processes, the relaxation time suggested by Herring for each normal process can be written as [32]:

$$
\left(\tau_{N}^{L}\right)^{-1}=C_{L} \omega^{2} T^{3} ;\left(\tau_{N}^{T}\right)^{-1}=C_{T} \omega T^{4}
$$

Where the values of the constants $C_{i}$ can be described in terms of the physical properties of the material, which according to the approximation made by Asen-Palmer et al. [66], take the form:

$$
C_{L} \approx \frac{K_{B}^{3} \gamma_{L}^{2} V}{M \hbar^{2} v_{L}^{5}} ; C_{T} \approx \frac{K_{B}^{4} \gamma_{T}^{2} V}{M \hbar^{3} v_{T}^{5}}
$$

where $\mathrm{V}$ is the unit cell volume.

\subsubsection{Isotope scattering}

The impurities are centers for phonon scattering. This mechanism is described as the isotope scattering and is related to the mass difference between the host atoms and the impurity atoms. Even in the case of pure crystals, without impurities, is necessary to consider the existence of isotopes, which efficiently scatter high frequency phonons. The perturbation in the lattice due to a substitutional atom was evaluated by Klemens using perturbation theory $[44,64]$, and the expression for the relaxation time due to isotope mixing can be expressed as

$$
\left(\tau_{I}^{i}\right)^{-1}=\frac{V \Gamma}{4 \pi v_{i}^{3}} \omega^{4} T^{4}
$$


Where $\Gamma$ is the mass-fluctuation parameter defined for a single element as $\Gamma=\sum_{i} f_{i}\left(1-\frac{M_{i}}{M}\right)^{2}$ where $f_{i}$ is the fractional atomic abundance of the isotope (impurity) of atomic mass $M_{i}[44]$.

\subsubsection{Boundary scattering}

A real crystal has a finite size; therefore, the scattering due to the crystal boundaries cannot be neglected. The boundaries act as highly efficient scattering regions, confining the MFP of the phonons to the order of the dimension of the sample. This problem was discussed by Ziman on the basis of the Casimir theory [28], and for the nanoscaled samples is the dominant scattering mechanism accounting for the reduction of the thermal conductivity. This process can be phenomenologically explained by considering two types of phonon boundary scattering processes; one of them corresponding to the specular phonon scattering, which mainly happens in smooth surfaces; while the other one corresponds to the pure diffusive phonon scattering, which mainly occurs on rough surfaces. Taking account of both processes, which occur with a probability $p$ for the pure specular scattering, and 1- $p$ for purely diffusive scattering, Ziman derived an expression for the MFP of phonons in a finite sample as $\Lambda=\frac{1+p}{1-p} \Lambda_{0}$ where $\Lambda_{0}$ is the MFP for a perfectly rough boundary [28], where only diffusive processes occur. It depends on the geometry of the sample; in the case of a cylinder, this MFP is equal to the diameter of the cylinder, $\Lambda_{0}=D$.

The key issue of this scattering lies in the form of the probability function, $p$, which has been addressed in different ways in the literature. Commonly, it has been taken equal to zero [25, $30,33,37]$ or as a constant independent of temperature and frequency [27, 34, 35], thereby simplifying the boundary scattering relaxation time. However, a more realistic approximation can be achieved if one eliminates such oversimplifications. Ziman showed that the probability function $p$ depends on the wavelength of the incident phonons [28], being the surface characterized by the mean value of the height deviation over a surface reference level; this parameter is called the asperity parameter, $\eta$. In a first approximation, the probability function can be written as $p=\exp \left[-{ }^{16 \pi^{3} \eta^{3}} / \lambda^{2}\right]$, which has been used by some authors [50]. Despite being a more accurate way of treating the boundary scattering with respect to the assumption of a constant value of $p$, the hypotheses assumed in the construction of this probability function were considered by Ziman asnon very realistic. Therefore, Ziman himself developed an alternative treatment, which takes account of the existence of a distribution function for the roughness instead of the average value given by the asperity parameter. In this way, the probability function takes the form $p(\eta, \lambda)=\int P(\eta) \operatorname{Exp}\left[-16 \pi^{3} \eta^{2} / \lambda^{2}\right] d \eta$ where $P(\eta)$ is the roughness distribution probability. This expression can be simplified if one considers that the reflection probability is a measure of the fraction of area whose average roughness is less than ${ }_{\pi 4}[28]$, reducing the probability 
function to $p(\eta, \lambda) \approx \int_{0}^{\lambda / 4 \pi} P(\eta) d \eta$. This is the form used by Mingo et al. for approaching the thermal conductivity in the low temperature range for rough Silicon NWs with a roughness distribution given as $P(\eta)=\frac{1}{\eta} \operatorname{Exp}\left[-\eta / \eta_{0}\right][67]$.

Moreover, based on the dependence of the probability function $p$ with frequency and roughness, Soffer addressed a different solution in the presence of a temperature gradient along the sample [68], according to which the probability function takes the form:

$$
p(\omega, \eta)=\operatorname{Exp}\left[-\left(2 \eta\left(\omega / v_{i}\right) \sin \theta\right)^{2}\right]
$$

where $\eta$ is the asperity parameter defined by Ziman, and $\theta$ the angle of incidence of phonons. This form given by Soffer is the one used in our simulations because we tested that it was the one providing the best results. Finally, adding the limitation in length to the boundary relaxation time, the final expression for the boundary relaxation time can be written as

$$
\left(\tau_{B}^{i}\right)^{-1}=\left(\frac{1-p}{1+p} \Lambda_{0}^{-1}+L^{-1}\right) v_{i}
$$

\subsection{The thermal conductivity of NWs}

Using this relaxation time, the thermal conductivity can be calculated over the full temperature range. The solution of the complex equation 16 was carried out by the Gauss-Kronrod local adaptive method, fixing a tolerance $<10^{-9}$ for enhancing the accuracy. This method provides calculation several times faster than other common numerical methods applied to this kind of problems. In order to test the model, first we carried out a prediction of the thermal conductivity of bulk silicon and germanium, obtaining an excellent fit to the experimental measurements over the full temperature range (Figure 5-A and 5-B).

The parameters used for the calculations are given in table 1 . The values for $\Gamma$ and $\gamma_{i}$ were taken from reference [61]; the Grüneisen parameter, $\gamma_{i}$, was approximated to a constant value. Actually, this is crude approach, since the Grüneisen parameter has a dependence with temperature, especially in the low temperature range [44]. The rough simplification of a constant value for this parameter arises from the dependence with the temperature and frequency of the three phonon scattering relaxation times, which is generically expressed as $\tau_{j}^{-1}=C t s_{j} \times G_{j}(\omega) \times F_{j}(T)$, namely the product of some function of $\omega$, some function of the temperature $\mathrm{T}$ and a given constant value for each type of scattering, $\mathrm{Ct} \mathrm{s}_{j}$. Therefore, the temperature dependence of each three-phonon scattering process can be completely included in 
the function $F(T)$; which encloses the temperature dependence of the Grüneisen parameter, while the constant parts of the Grüneisen parameters are encompassed in the constant group $C t s_{j}$. The bulk value for the sound velocities of each phonon branch was taken from [44]. Finally, the asperity parameter was chosen assuming a smooth surface, matching the values reported in [40] for smooth silicon NWs.
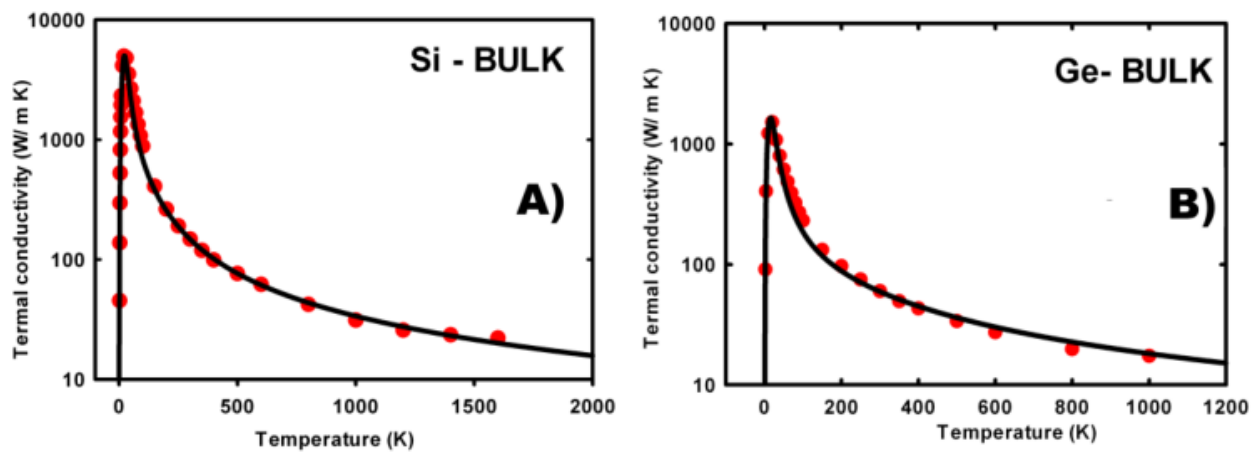

Figure 5. Fitting of the experimental thermal conductivities of bulk Si (A), and bulk Ge(B) by our modified CallawayHolland model. Experimental data taken from [14].

The values of the cut-off temperatures, $\theta_{i}$, and the effective size of the sample remains as free parameters in the resolution of the equations. The effective size is characterized by the lengths $\mathrm{L}$ and $\Lambda_{0}$, which take the same value as in the bulk for a sake of simplicity.

\begin{tabular}{ccccccccccc}
\hline & $\mathrm{L}(\mathbf{m})$ & $\boldsymbol{\Lambda}_{\mathbf{0}}(\mathbf{m})$ & $\boldsymbol{\eta}(\mathbf{m})$ & $\boldsymbol{\Gamma}$ & $\boldsymbol{\theta}_{\boldsymbol{L}}$ & $\boldsymbol{\theta}_{\boldsymbol{T}}$ & $\boldsymbol{v}_{\boldsymbol{L}}$ & $\boldsymbol{v}_{\boldsymbol{T}}$ & $\boldsymbol{V}_{\boldsymbol{L}}$ & $\boldsymbol{V}_{\boldsymbol{T}}$ \\
\hline $\mathrm{Si}$ & $6 \times 10^{-3}$ & $6 \times 10^{-3}$ & $1 \times 10^{-9}$ & $2.16 \times 10^{-4}$ & 627.7 & 435.8 & 8430 & 5840 & 1.1 & 0.66 \\
\hline $\mathrm{Ge}$ & $4 \times 10^{-3}$ & $4 \times 10^{-3}$ & $1 \times 10^{-9}$ & $6.08 \times 10^{-4}$ & 387.1 & 278.3 & 4920 & 3540 & 1.1 & 0.66 \\
\hline
\end{tabular}

Table 1. Parameters used in the calculations of the thermal conductivity of bulk Si and bulk Ge.

The equivalent cut-off temperature obtained as $\theta=3\left(\theta_{L}^{-1}+2 \theta_{T}^{-1}\right)^{-1}$ [69] has a value of $495 \mathrm{~K}$ for $\mathrm{Si}$, and $307 \mathrm{~K}$ for $\mathrm{Ge}$. In the original Callaway model the cut-off temperature matches the Debye temperature, albeit this parameter has been treated in different ways in further works. The Debye temperature for Si determined from the specific heat takes a value of 645 $\mathrm{K}$ [61]. If one uses the sound velocities of the acoustic branches instead of the heat capacity, the cut-off temperature scales up to $710 \mathrm{~K}$ [61]; whereas, if one estimates this temperature through the zone-boundary frequencies of the transversal and longitudinal acoustic phonons, the value drops dramatically to $298 \mathrm{~K}$ [61]. One can also consider the fact that the Callaway model ignores the contributions of optical phonons, because of the continuum approximation. Fortunately, this limitation can be circumvented by increasing the cut-off 
temperatures [44]. Thus, the use of the cut-off temperature as a free adjustable parameter implicitly includes the optical phonon contribution.

Finally, the equivalent sample diameter, being of the order of millimeters, does not have a big impact in the thermal conductivity for temperatures above $300 \mathrm{~K}$. This allows to adjust the temperature cut-offs for matching the experimental values in this temperature range. Therefore, the equivalent sample diameter is commonly used to matching the thermal conductivity in the low temperature range [61].

On the other hand, one can assume that the phonon confinement effects only show relevant effects below $25 \mathrm{~nm}$ in diameter [15]; this means that one can treat the NWs with diameter larger than $25 \mathrm{~nm}$ in a bulk-like approach.

Having established the excellent agreement with the experimental data for bulk Si and Ge, the same procedure was applied to Si NWs, and the results were compared to those measured in [11]. The parameters used for these calculations are given in Table 2. The agreement with the experimental results was excellent over the full temperature range for NWs without phonon confinement ( $\mathrm{D}>25 \mathrm{~nm})$. While the results, as expected, show imprecise below the phonon confinement threshold $(\mathrm{D}<25 \mathrm{~nm})$. The pseudo-linear experimental behavior of the $22 \mathrm{~nm}$ NW cannot be predicted by this bulk-like model; which suggests that alternative approaches such as the one conducted in [36] are required for describing the thermal transport in NWs of reduced diameter, exhibiting confinement effects. These results are summarized in Figure 6.

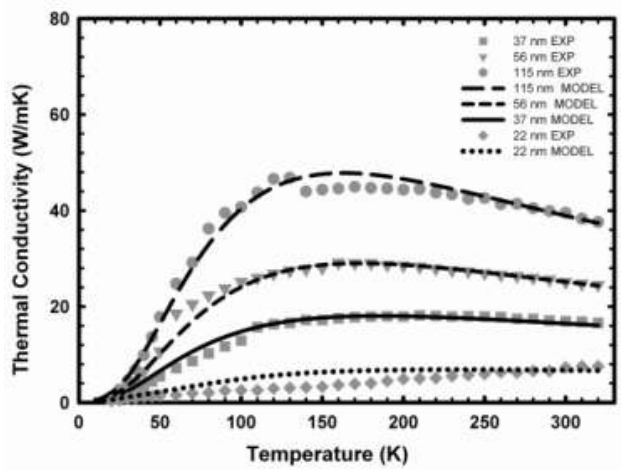

Figure 6. Fitting to the experimental thermal conductivity of Si NWs with different diameters, showing the excellent fit for all of them, except for the $22 \mathrm{~nm} \mathrm{NW}$. Experimental data taken from [11]

Several authors have shown that the speed of sound of the different phonon branches is lower in semiconductor NWs than in their corresponding bulk counterparts, even for NWs with diameters larger than the one for phonon confinement [25, 27, 34, 40,69]. The values of the sound velocities present differences depending upon the method followed to obtaining them; therefore, the sound velocities are left as free parameters when adjusting the experi- 
mental thermal conductivity data. The cut-off temperatures for semiconductor NWs must be also lower than their bulk counterparts [30, 34, 40,69]. This is consistent with the values of the cut-off temperatures and the sound velocities found by fitting the experimental thermal conductivity data, Table 2.

\begin{tabular}{|c|c|c|c|c|c|c|c|c|c|c|}
\hline NW & $L(m)$ & $\Lambda_{0}(m)$ & $\eta(\mathrm{m})$ & $r$ & $\theta_{L} \quad(\mathrm{~K})$ & $\theta_{T}(\mathrm{~K})$ & $v_{L}(\mathrm{~m} / \mathrm{s})$ & $v_{T}(\mathrm{~m} / \mathrm{s})$ & $v_{L}$ & $\gamma_{T}$ \\
\hline 115 & $5 \times 10^{-9}$ & $115 \times 10^{-9}$ & $1 \times 10^{-9}$ & 2. $16 \times 10^{-4}$ & 537.5 & 302.9 & 7962 & 4741 & 1.1 & 0.66 \\
\hline 56 & $5 \times 10^{-9}$ & $56 \times 10^{-9}$ & $1 \times 10^{-9}$ & 2. $16 \times 10^{-4}$ & 511.1 & 284.1 & 7168 & 4163 & 1.1 & 0.66 \\
\hline 37 & $5 \times 10^{-9}$ & $37 \times 10^{-9}$ & $1 \times 10^{-9}$ & 2. $16 \times 10^{-4}$ & 479. 4 & 259.3 & 7066 & 4106 & 1. 1 & 0.66 \\
\hline 22 & $5 \times 10^{-9}$ & $22 \times 10^{-9}$ & $1 \times 10^{-9}$ & 2. $16 \times 10^{-4}$ & 466.9 & 141.2 & 7018 & 3997 & 1. 1 & 0.66 \\
\hline
\end{tabular}

Table 2. Parameters used in the calculations of the thermal conductivity of smooth Si NWs of [11].

On the other hand, if one assumes that the crystallinity and the impurity concentration are the same for rough and smooth NWs, the only relevant difference between both types of NWs is the surface roughness; therefore, the thermal conductivity reduction in rough NWs must be exclusively related to the contribution of the roughness to the boundary scattering. The roughness is included in the boundary scattering through the asperity parameter $\eta$; choosing a value of $\eta=1 \mathrm{~nm}$, typically reported for smooth NWs [40], the value of $p$ is close to zero for most of the phonon frequencies, which means that even for such small roughness the boundary scattering is close to the diffusive limit in agreement with the result of reference [56]. Therefore, further increase of $\eta$ cannot result in a substantial increase of the diffusive scattering contribution, and the dramatic reduction of the thermal conductivity, experimentally observed in rough NWs [2], cannot be accounted for by the alone diffusive boundary scattering. The breakdown of the Casimir limit observed in [57] allows us to introduce in the calculation the concept of an effective Casimir length $\left(\Lambda_{0}^{\prime}<\Lambda_{0}\right)$ smaller than the classical MFP given by the diameter of the NW consequence of the shrinkage of the acoustic phonon MFP in rough NW. The prediction of the thermal conductivity of rough NWs requires the evaluation of the effective Cassimir length $\Lambda_{0}^{\prime}$; for this purpose we used the experimental data of a Si NW with $115 \mathrm{~nm}$ diameter and roughness of $\eta=5.7 \mathrm{~nm}$ [2]. Assuming that the only difference with the smooth NW of the same diameter lies on the roughness, all the other parameters showed in Table 2 already used for the calculation of the thermal conductivity of the smooth NW of the same dimension $(115 \mathrm{~nm})$ remained unchanged. In Figure 7-A the thermal conductivity calculated for the rough NW with an effective Casimir length $\Lambda_{0}^{\prime}=115 \mathrm{~nm}$ gives a value for the thermal conductivity at $300 \mathrm{~K}$ only $1 \mathrm{~W} / \mathrm{mK}$ less than the thermal conductivity of the 115 smooth NW; therefore, the very increase of the asperity parameter cannot account for the abrupt decrease of the thermal conductivity in rough NWs, as long as the phonon MFP is not reduced below the Casimir limit; however, as the MFP is reduced, the thermal conductivity falls down dramatically, reaching the experimental value reported in [2] for an effective Cassimir length of $\Lambda_{0}^{\prime} \approx 13 \mathrm{~nm}$, Figure 7-A. 

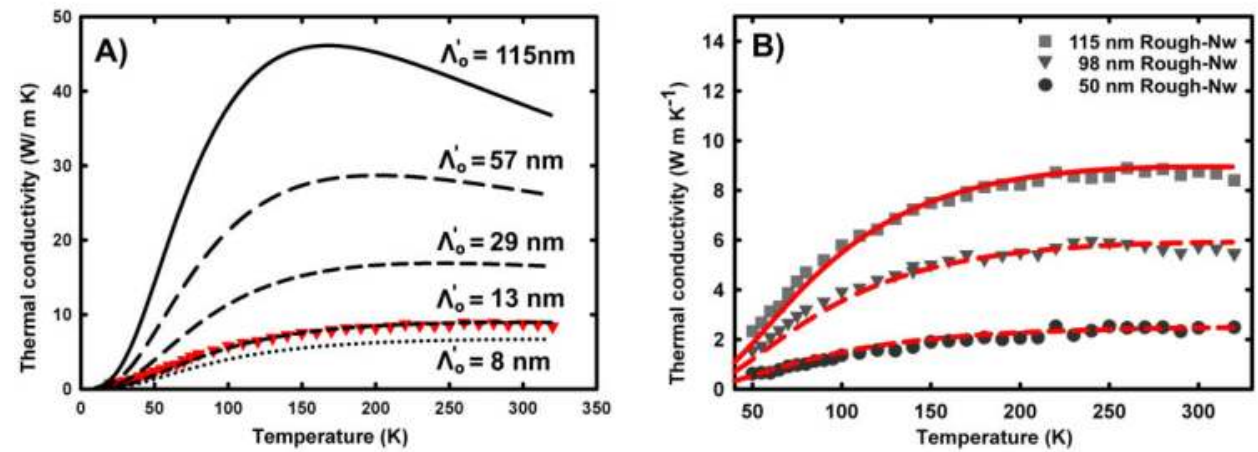

Figure 7. Calculated thermal conductivities of a rough Si NW ( $115 \mathrm{~nm}$ diameter) for different effective Casimir lengths, showing fitting to experimental data ( taken from [2]) for $\Lambda_{0}^{\prime}=13 \mathrm{~nm}(A)$; fitting of the experimental thermal conductivities for rough Si NWs with different diameters, the fitting parameters are summarized in Table. 3 (B), experimental data taken from [2].

This large MFP reduction in rough NWs cannot be explained in terms of the surface scattering mechanism described by Ziman [28], and must be considered in a first instance as an admissible calculation tool for obtaining good experimental data approach as was shown in [17]. The thermal conductivities calculated for NWs with the same geometric characteristics as those measured in [2] are shown in Figure 7-B, evidencing a very good fitting to the experimental data, using the parameters listed in Table 3. The effective Casimir lengths obtained by fitting the experimental values show a significant reduction with respect to the NW diameters, the ideal Casimir lengths; similar reductions have been also reported by other authors [17].

\begin{tabular}{cccccccccc}
\hline $\mathrm{D}(\mathrm{nm})$ & $\mathrm{L}(\mathrm{m})$ & $\boldsymbol{\Lambda}_{\mathbf{0}}(\mathrm{m})$ & $\boldsymbol{\eta}(\mathrm{m})$ & $\boldsymbol{\theta}_{\boldsymbol{L}}(\mathrm{K})$ & $\boldsymbol{\theta}_{\boldsymbol{T}}(\mathrm{K})$ & $\boldsymbol{v}_{\boldsymbol{L}}(\mathrm{m} / \mathrm{s})$ & $\boldsymbol{v}_{\boldsymbol{T}}(\mathrm{m} / \mathrm{s})$ & $\boldsymbol{\gamma}_{L}$ & $\boldsymbol{\gamma}_{\boldsymbol{T}}$ \\
\hline 115 & $5 \times 10^{-9}$ & $13 \times 10^{-9}$ & $5.7 \times 10^{-9}$ & 537.5 & 302.9 & 7962 & 4741 & 1.1 & 0.66 \\
\hline 98 & $5 \times 10^{-9}$ & $8.8 \times 10^{-9}$ & $5.7 \times 10^{-9}$ & 530.1 & 300.2 & 7745 & 4584 & 1.1 & 0.66 \\
\hline 50 & $5 \times 10^{-9}$ & $3.3 \times 10^{-9}$ & $5.7 \times 10^{-9}$ & 505.9 & 274 & 7159 & 4160 & 1.1 & 0.66 \\
\hline
\end{tabular}

Table 3. Parameters used in the calculations of the thermal conductivity of rough NW of [2].

\subsection{A predictive expression for the thermal conductivity of Si NWs}

A big constraint for the applications of NWs in which the thermal conductivity plays a major role is the lack of experimental data over a large range of diameters and temperatures, or in its defect the absence of a model supplying such information. In view of the difficulties inherent to thermal conductivity measurements at the nanoscale, and the variety of diameters and surface roughness, the development of a model capable of predicting the thermal conductivity in a wide range of diameters and surface roughness acquires a capital interest. 
We have shown that one can achieve an excellent fit of the experimental thermal conductivity of semiconductor NWs using a modified Callaway-Holland formalism. This agreement should permit us the prediction of the thermal conductivity for NW diametersand the temperature ranges for which experimental data are not available. Particularly relevant is the case of the high temperature range, above $400 \mathrm{~K}$, for which there are not experimental measurements available. Since the model for bulk Si provides a very good result in the high temperature range, it is reasonable to assume that the same model for NWs without phonon confinement works well in the high temperature range.

In our model, the fitting to the thermal conductivity is achieved using the speeds of sound and the cut-off temperatures for transverse and longitudinal modes, as free parameters. By relating those parameters to the NW diameter, one could predict the thermal conductivity for any NW diameter of a given roughness. In the case of the smooth NWs, a very good result was obtained for NWs with a diameter above the phonon confinement limit. Unfortunately, there are only three diameters for which reliable experimental data are available. However, additional data exist when one considers rough NWs; assuming that the reduction in the thermal conductivity of these NWs with respect to smooth NWs is exclusively related to the contribution of the roughness to the boundary scattering, which does not modify the sound velocities, nor the cut-off temperatures, one can extend the range of available experimental data. This hypothesis was confirmed with rough NWs of $115 \mathrm{~nm}$ diameter, for which an excellent fitting was achieved by acting only on the boundary relaxation time, keeping all the other parameters the same as the ones used for the smooth $115 \mathrm{~nm} \mathrm{NW}$ (see section 4. 4.). Therefore, one can use the values of the adjustable parameters, speed of sound, and cut off-temperature, of both smooth and rough NWs to build up the predictive expression for the thermal conductivity.

The values of the speed of sound and cut-off temperature estimated for each NW diameter for which experimental data are available are plotted in Figure 8 (dots). In this figure one can observe that the behavior of the both velocities, the speed of sound of the longitudinal acoustic branch and the transverse acoustic branch respectively, have the same smooth form. Similar behavior is observed for the cut-off temperatures for each branch. This allows us to assume that those free parameters can be phenomenologically expressed as a function of the NW diameter. In the same figure, we show the nonlinear regression for each free parameter, showing in all the cases an adjustment of $r^{2}>0.99$ to a generic function expressed as

$$
f(D)=\frac{1+a D}{b+c D}
$$

Therefore, with these phenomenological relations one is allowed to interpolate the values of the free parameters used in the calculation of the thermal conductivity of Si NWs in a range of diameters between the phonon confinement limit to slightly above the hundred of nms in diameter, using for that the adjustment parameters given in Table 4. Note that these parameters only represent numbers suitable for describing the sound velocities and the temperature cut-offs as a function of the NW diameter. 

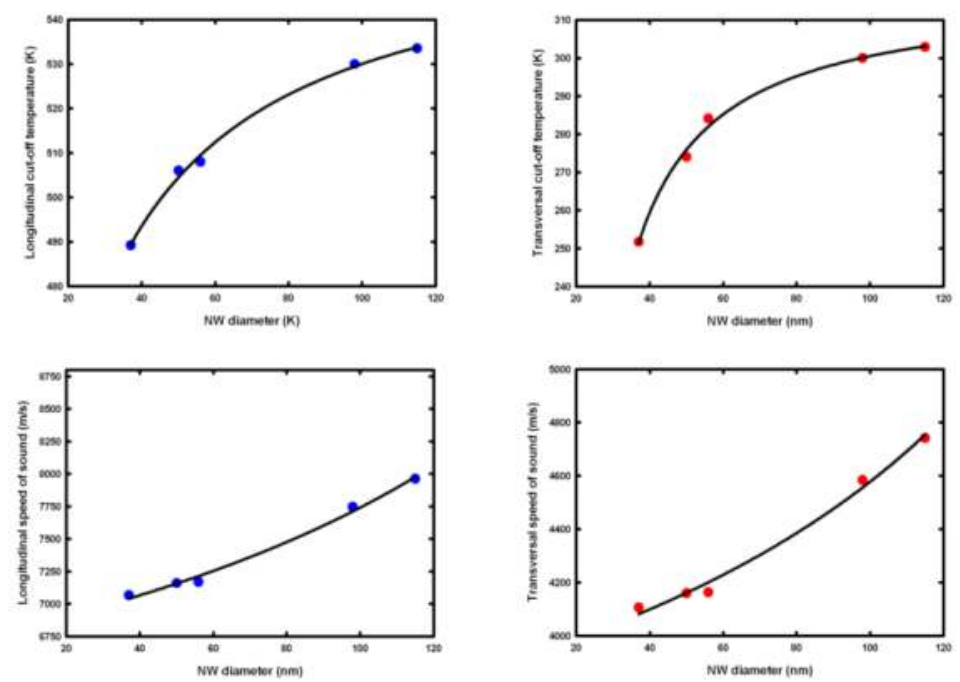

Figure 8. Plots of the cut-off temperatures, and sound speeds for the longitudinal and acoustic phonon branches vs the NW diameter. The symbols represent the values obtained by fitting the available experimental data. The lines are the fits using the phenomenological three parameter equation 25 in the text body.

\begin{tabular}{cccc}
\hline & $\mathbf{a}$ & $\mathbf{b}$ & $\mathbf{c}$ \\
\hline$v_{L}$ & $-2,362 \times 10^{-3}$ & $1,478 \times 10^{-4}$ & $-4,91310^{-7}$ \\
\hline$v_{T}$ & $-2,563 \times 10^{-3}$ & $2,566 \times 10^{-4}$ & $-9,41410^{-7}$ \\
\hline$\theta_{L}$ & $1,668 \times 10^{-1}$ & $3,671 \times 10^{-3}$ & $2,969 \times 10^{-4}$ \\
\hline$\theta_{T}$ & $-5,153 \times 10^{-2}$ & $2,395 \times 10^{-3}$ & $-1,621 \times 10^{-4}$ \\
\hline
\end{tabular}

Table 4. Parameter values from the non linear regressions to the data of Figure 8.

Using these parameters one can construct the thermal conductivity vs T plots for any NW diameter. These plots are shown in Figure 9-A. The thermal conductivity vs the NW diameter for two fixed temperatures, $300 \mathrm{~K}$ and $600 \mathrm{~K}$, are represented in Figure 9-B; one observes that at high temperature the influence of the diameter on the thermal conductivity is flattened out with respect to what happens at lower temperatures; which roughly can be interpreted in terms of the balance between boundary scattering processes and three phonon processes. In the low temperature range $(\mathrm{T}<300 \mathrm{~K})$ the boundary scattering is dominant, while in the high temperature range the three phonon scattering processes increase its weight. 

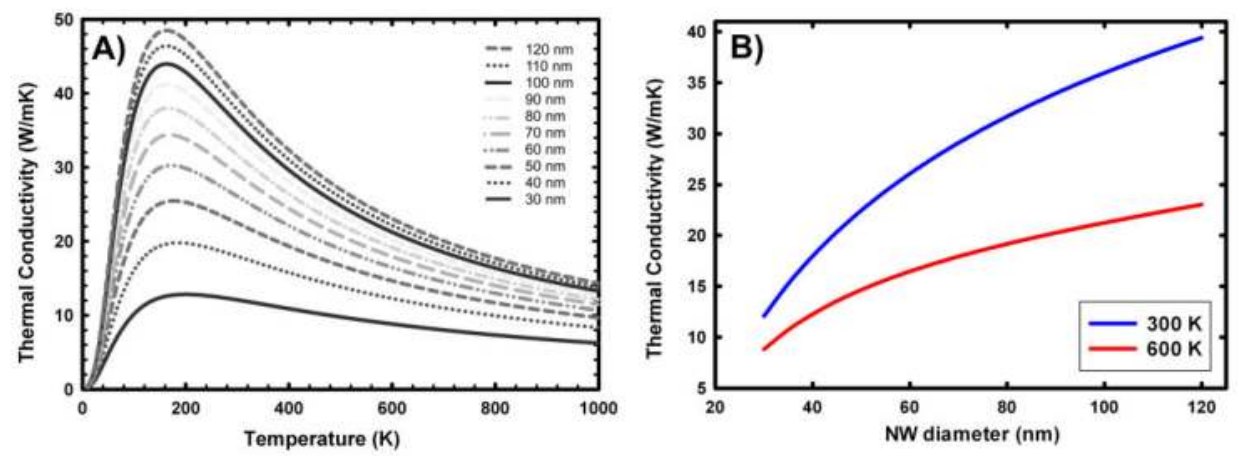

Figure 9. Calculated thermal conductivity for arbitrary diameters using the parameters given in Table 3 (A). Thermal conductivities at $300 \mathrm{~K}$ and $600 \mathrm{~K}$ as a function of the NW diameter (B)

\section{Summary and perspectives}

An overview of the thermal transport in semiconductor NWs has been presented. Both experimental and theoretical aspects have been discussed, evidencing that the theoretical models need of reliable thermal conductivity measurements to contrast the models, in order to advance in the understanding of the thermal transport in semiconductor NWs. Several clues need to be addressed in the close future; in particular, the role of optical phonons scattering, the scattering mechanisms in low diameter NWs, in connection with the phonon confinement phenomena; and a comprehensive view of the boundary scattering in rough surfaces, for which a breakdown of the Casimir limit occurs; also, the modeling of the thermal transport in alloyed NWs has to be addressed because of the importance of alloys for engineering the thermal conductivity. We have developed a model based on a modified Callaway-Holland formalism, which agglomerates the contributed ideas provided by different authors. This model permits an excellent fitting of the experimental results over an extended temperature range. Using the fitting of this model to the available experimental results one established empiric relations that permit to build up a predictive tool, which opens the access to the thermal conductivity of any Si NW diameter above the phonon confinement limit. This formalism can be extended to any other semiconductor without loss of generality, provided that reliable experimental thermal conductivity data for a few NWs diameters are available.

\section{Acknowledgement}

This work was funded by the Spanish Government (MAT-2007-66181-C03 and MAT-2010-20441-C02) 


\section{Author details}

J. Anaya ${ }^{1}$, J. Jiménez ${ }^{1}$ and T. Rodríguez ${ }^{2}$

1 GdS Optronlab, Ed I+D, University of Valladolid, Valladolid, Spain

2 Electronic Technology, ETSIT, Polytechnic University of Madrid, Madrid, Spain

\section{References}

[1] Cui Y, Lieber C.M. Functional Nanoscale Electronic Devices Assembled using Silicon Nanowire Building Blocks. Science 2001;291, 851.

[2] Hochbaum A.I, Chen R, Delgado R.D, Liang W, Garnett E.C, Najarian M, Majumdar A, Yang P. Rough Silicon Nanowires as High Performance Thermoelectric Materials. Nature 2008;451, 163.

[3] Kayes B.M, Atwater H.A, Lewis N.S. Comparison of the device physics principles of planar and radial p-n junction nanorod solar cells. J. Appl. Phys. 2005;97, 114302.

[4] Xie X.Q, Liu W.F, Oh J.I, Shen W.Z. Optical absorption in c-Si/a-Si:H core/shell nanowire arrays for photovoltaic applications. Appl. Phys. Lett. 2011;99, 033107.

[5] Singh N, Buddharaju K.D, Manhas S.K, Agarwal A, Rustagi S.C, Lo G.Q, Balasubramanian N, Kwong D.L. Si, SiGe Nanowire Devices by Top-Down Technology and Their Applications. IEEE Trans. Electron Devices 2008;55, 3107.

[6] Westover T, Jones R, Huang J.Y, Wang G, Lai E, Talin A.A. Photoluminescence, Thermal Transport, and Breakdown in Joule-Heated GaN Nanowires. Nano Lett. 2009;9, 257.

[7] Shi L, Yao D, Zhang G, Li B. Large thermoelectric figure of merit in Si1-xGex nanowires. Appl. Phys. Lett. 2010;96, 173108.

[8] Mingo N. Thermoelectric figure of merit of II-VI semiconductor nanowires. Appl. Phys. Lett. 2004;85, 5986.

[9] Mingo N. Thermoelectric figure of merit and maximum power factor in III-V semiconductor nanowires. Appl. Phys. Lett. 2004;84, 2652.

[10] Shi L, Li D, You C, Jang W, Kim D, Yao Z, Kim P, Majumdar A. Measuring Thermal and Thermoelectric Properties of One-Dimensional Nanostructures Using a Microfabricated Device. J. Heat Transfer 2003;125, 881.

[11] Li D, Wu Y, Kim P, Shi L, Yang P, Majumdar A. Thermal conductivity of individual silicon nanowires. Appl. Phys. Lett. 2003;83, 2934. 
[12] Hippalgaonkar K, Huang B, Chen R, Sawyer K, Ercius P, Majumdar A. Fabrication of Microdevices with Integrated Nanowires for Investigating Low-Dimensional Phonon Transport. Nano Lett. 2010;10 4341.

[13] Li D.Y, Wu Y, Fan R, Yang P.D, Majumdar A. Thermal conductivity of Si/SiGe superlattice nanowires. Appl. Phys. Lett. 2003;83, 3186.

[14] Glassbrenner J.C, Slack G.A. Thermal Conductivity of Silicon and Germanium from $3^{\circ} \mathrm{K}$ to the Melting Point. Phys. Rev. 1964;134, 4A 1058.

[15] Campbell I. H, Fauchet P.M. The effects of microcrystal size and shape on the one phonon Raman spectra of crystalline semiconductors. Solid St. Commun. 1986;58, 739.

[16] Zhou J, Jin C, Seol J.H, Li X, Shi L. Thermoelectric properties of individual electrodeposited bismuth telluride nanowires. Appl. Phys. Lett 2005;87, 133109.

[17] Guthy C, Nam C, Fischer J.E. Unusually low thermal conductivity of gallium nitride nanowires. J. Appl. Phys. 2008;103, 064319.

[18] You C. Large Thermoelectric Figure-of-Merits from SiGe Nanowires by Simultaneously Measuring Electrical and Thermal Transport Properties. Nano Lett. 2012;12, 2918.

[19] Shi L, Hao Q, Choongho Y, Mingo N, Xiangyang K. Thermal conductivities of individual tin dioxide nanobelts. Appl. Phys. Lett. 2004;84, 2638.

[20] Wingert M.C, Chen Z.C.Y, Dechaumphai E, Moon J, Kim J, Xiang J, Chen R. Thermal Conductivity of Ge and Ge-Si Core-Shell Nanowires in the Phonon Confinement Regime. Nano Lett 2011;11, 5507.

[21] Park Y, Kim J, Kim H, Kim I, Lee K, Seo D, Choi H, Kim W. Thermal conductivity of VLS-grown rough $\mathrm{Si}$ nanowires with various surface roughnesses and diameters. Appl. Phys. A 2011;104,7.

[22] Lim J, Hippalgaonkar K, Andrews S.C, Majumdar A, Yang P. Quantifying Surface Roughness Effects on Phonon Transport in Silicon Nanowires. Nano Lett. 2012;12, 2475 .

[23] Kim H, Kim I, Choi H, Kim W. Thermal conductivities of Si1-xGex nanowires with different germanium concentrations and diameters. Appl. Phys. Lett. 2010;96, 233106.

[24] Kim H, Park Y, Kim I, Kim J, Choi H, Kim W. Effect of surface roughness on thermal conductivity of VLS-grown rough Si1-xGex nanowires. Appl. Phys. A 2011;104, 23.

[25] Balandin A, Wang K.L. Significant decrease of the lattice thermal conductivity due to phonon confinement in a free-standing semiconductor quantum well. Phys. Rev B $1998 ; 58,1544$.

[26] Parrott J. E., Stuckes A. D. Thermal Conductivity of Solids, New York: Methuen; 1975. 
[27] Zou J, Balandin A. Phonon heat conduction in a semiconductor nanowire. J. Appl. Phys. 2001;89, 2932.

[28] Ziman J.M. Electrons and Phonons New York: Oxford University Press; 1967.

[29] Volz S.G, Chen G. Molecular dynamics simulation of thermal conductivity of silicon nanowires. Appl. Phys. Lett. 1999;75 2056.

[30] Mingo N. Calculation of Si nanowire thermal conductivity using complete phonon dispersion relations. Phys. Rev. B 2003;68, 113308.

[31] Mingo N, Yang L. Predicting the Thermal Conductivity of Si and Ge Nanowires. Nano Lett. 2003;3, 1713.

[32] Herring C. Role of Low-Energy Phonons in Thermal Conduction. Phys. Rev 1954;95, 954.

[33] Chung J.D, McGaughey A.J.H, Kaviany M. Role of Phonon Dispersion in Lattice Thermal Conductivity Modeling. Transactions of the ASME 2004;126, 376.

[34] Huang M, Chong W, Chang T. The lattice thermal conductivity of a semiconductor nanowire. J. Appl. Phys. 2006;99 114318.

[35] Liang L.H, Li B. Size-dependent thermal conductivity of nanoscale semiconducting systems. Phys. Rev. B 2006;73, 153303.

[36] Murphy P.G, Moore J.E. Coherent phonon scattering effects on thermal transport in thin semiconductor nanowires. Phys. Rev. B 2007;76, 155313.

[37] Tian Z, Esfarjani K, Shiomi J, Henry A.S, Chen G. On the importance of optical phonons to thermal conductivity in nanostructures. Appl. Phys. Lett 2011;99, 053122.

[38] Ward A, Broido D.A. Intrinsic phonon relaxation times from first-principles studies of the thermal conductivities of Si and Ge. Phys. Rev B 2010;81, 085205.

[39] Kazan M, Pereira S, Coutinho J, Correia M.R, Masri P. Role of optical phonon in Ge thermal conductivity. Appl. Phys. Lett 2008;92, 211903.

[40] Kazan M, Guisbiers G, Pereira S, Correia M, Masri P, Bruyant A, Volz S, Royer P. Thermal conductivity of silicon bulk and nanowires: Effects of isotopic composition, phonon confinement, and surface roughness. J. Appl. Phys 2010;107, 083503.

[41] Callaway J. Model for Lattice Thermal Conductivity at Low Temperatures. Phys. Rev. 1959;113, 1046.

[42] Holland M.G. Analysis of Lattice Thermal Conductivity. Phys. Rev 1963;132, 2461.

[43] Carruthers P. Theory of Thermal Conductivity of Solids at Low Temperatures. Rev. Mod. Phys 1961;33, 92.

[44] Srivastava G.P. The Physics of Phonons, Bristol:Adam Hilger; 1990. 
[45] Alvarez F.X, Jou1 D. Size and frequency dependence of effective thermal conductivity in nanosystems. J. Appl. Phys, 2008;103, 094321.

[46] Guyer R.A, Krumhansl J.A. Solution of the Linearized Phonon Boltzmann Equation. Phys. Rev 1966;148, 766.

[47] Guyer R.A, Krumhansl J.A. Thermal Conductivity, Second Sound, and Phonon Hydrodynamic Phenomena in Nonmetallic Crystals. Phys. Rev 1966;148, 778.

[48] Alvarez F.X., Jou D, Sellitto A. Phonon hydrodynamics and phonon-boundary scattering in nanosystems. J. Appl. Phys, 2009;105, 014317.

[49] Sellitto A. Alvarez F.X, Jou D. Second law of thermodynamics and phonon-boundary conditions in nanowires. J. Appl. Phys. 2010;107, 064302.

[50] Moore A.L, Saha S.K, Prasher R.S, Shi L. Phonon backscattering and thermal conductivity suppression in sawtooth nanowires. Appl. Phys. Lett 2008;93, 083112.

[51] Sellito A, Alvarez F.X, Jou D., Temperature dependence of boundary conditions in phonon hydrodynamics of smooth and rough nanowires. J. Appl. Phys 2010;107, 114312.

[52] Martin P, Aksamija Z, Pop E, Ravaioli U. Impact of Phonon-Surface Roughness Scattering on Thermal Conductivity of Thin Si Nanowires. Phys. Rev. Lett. 2009;102, 125503.

[53] Kosevich Y.A, Savin A.V. Reduction of phonon thermal conductivity in nanowires and nanoribbons with dynamically rough surfaces and edges, EPL 2009;88, 14002.

[54] Liu L,Chen X. Effect of surface roughness on thermal conductivity of silicon nanowires. J. Appl. Phys 2010;107, 033501.

[55] Donadio D, Galli G. Atomistic Simulations of Heat Transport in Silicon Nanowires. Phys. Rev. Lett. 2009;102, 195901.

[56] Luisier M. Investigation of thermal transport degradation in rough Si nanowires. J. Appl. Phys 2011;110, 074510.

[57] Sadhu J, Sinha S. Room-temperature phonon boundary scattering below the Casimir limit. Phys. Rev. B 2011;84, 115450.

[58] Yang N, Zhang G, Li B. Ultralow Thermal Conductivity of Isotope-Doped Silicon Nanowires. Nano Lett. 2008;8, 276.

[59] Chen J, Zhang G, Li B. Tunable thermal conductivity of $\mathrm{Si}_{1-x} \mathrm{Ge}_{\mathrm{x}}$ nanowires. Appl.Phys.Lett 2009;95, 073117.

[60] Wang Z, Mingo N. Diameter dependence of SiGe nanowire thermal conductivity. Appl. Phys. Lett 2010;97, 101903. 
[61] Morelli D.T., Heremans J.P, Slack G.A. Estimation of the isotope effect on the lattice thermal conductivity of group IV and group III-V semiconductors. Phys. Rev B 202;66, 195304.

[62] Peierls R. E. Quantum Theory of Solids New York: Oxford University Press; 1955.

[63] Sharma P.C, Frank Rose M. Three-phonon scattering processes and their role in phonon thermal conductivity of silicon. Journal Solid State Chemistry 1998;73, 92.

[64] Klemens P.G. Solid State Physics, Vol 7, p.1 New York: Academic; 1958.

[65] Slack G. A. Thermal Conductivity of CaF2, MnF2, CoF2, and ZnF2 Crystals. Phys. Rev. 1961;122, 1451.

[66] Asen-Palmer M. Bartkowski K, Gmelin E, Carona M, Zhernov A.P, Inyushkin V.A, Taldenkov A, Ozhogin V.I, Itoh K.M, Haller E.E. Thermal conductivity of germanium crystals with different isotopic compositions. Phys. Rev. B 1997;56, 9431.

[67] Heron J. S, Fournier T, Mingo N, Bourgeois O. Mesoscopic Size Effects on the Thermal Conductance of Silicon Nanowire. Nano Lett. 2009;9, 1861.

[68] Soffer S.B. Statistical Model for the Size Effect in Electrical Conduction. J. Appl. Phys. $1967 ; 38,1710$.

[69] Omar M.S, Taha H.T. Lattice dislocation in Si nanowires. Physica B 2009;404, 5203. 